\title{
The role of technology in avoiding bias in the design and execution of clinical trials
}

\author{
This article was published in the following Dove Press journal: \\ Open Access Journal of Clinical Trials \\ 25 January 2013 \\ Number of times this article has been viewed
}

\section{Hazel Goodale \\ Damian McEntegart}

Perceptive Informatics Inc, Nottingham, United Kingdom

Correspondence: Hazel Goodale Perceptive Informatics Inc, Lady Bay House, Meadow Grove, Nottingham, NG2 3HF, United Kingdom

Tel +44 II 58443962

Fax +44 II5 9557555

Email hazel.goodale@perceptive.com

\begin{abstract}
There are many documented instances in which bias has had an adverse effect on the results of clinical trials. This has led to a number of design techniques being developed that can be implemented in clinical trials in order to reduce bias. Sources of bias referring to published case studies are reviewed and discussed. The potential uses of technology to alleviate bias are outlined, particularly the use of centralized interactive response systems to randomize patients and manage medication in such a way as to limit the risk of bias caused by knowledge of either a patient's current treatment or future treatment assignments. Potential sources of bias include selection bias, accidental bias, assessment bias, observer bias, and operational bias. These can arise through inadequate randomization and concealment methods during the trial. The blind may be broken by individual code breaks or through deduction in studies with frequent dose adjustments; there is scope for deduction in adaptive trials that might also introduce bias. Technology can reduce or eliminate the potential for bias in a variety of manners including central randomization and secure methods to protect the blinding and trial integrity. However, if the separation of randomization and dispensing, made possible by the use of technology, is not applied correctly then new unblinding scenarios can be introduced.
\end{abstract}

Keywords: electronic systems, IVR, blinding, randomization

\section{Introduction}

The International Conference on Harmonization (ICH) E9 Guidance ${ }^{1}$ notes that many of the principles outlined within the guidance are aimed at minimizing bias, which is defined as:

the systematic tendency of any factors associated with the design, conduct, analysis, and interpretation of the results of clinical trials to make the estimate of a treatment effect deviate from its true value. ${ }^{1}$

The Guidance also notes that the most important design techniques for avoiding bias are randomization and blinding. The use of technology to implement these two design techniques and thus reduce bias is one area addressed by this article; we also cover other uses of technology.

The CONSORT guidance ${ }^{2}$ divides randomization and blinding into subcomponents, which helps define the concepts. The components are the method used for generation of the random allocation sequence, the type of randomization including details of any restrictions, the mechanism used to implement the randomization, the acts of generation and treatment assignment, the question of who was blinded after assignment to treatment, and the description of the similarity of the interventions. 
In this article we outline five sources of bias - selection, accidental, assessment, observer, and operational. We give examples of how each may arise, their potential effects, and how the risk of their occurrence may be mitigated or even eliminated by the use of technology.

\section{Materials and methods}

In the sections that follow, we describe various sources of bias and how technology can be used to eliminate or reduce the risk of occurrence. We cite various references covering examples and the effects of bias. These references were identified by an automated monthly search of the PubMed database (http://www.ncbi.nlm.nih.gov/pubmed), conducted over a period of 10 years by one of the authors (DM). Twenty search terms were utilized to capture articles relevant to all aspects of randomization and blinding methodology in clinical trials; the list of terms included "bias" joined with the words "selection," "accidental," "assessment," and "operational."

\section{Selection bias}

If the investigator knows (or can guess with a success rate that is higher than might be expected through chance) which treatments will be allocated to future patients at their site, then the trial may be open to conscious or unconscious selection bias. The investigator may decide not to enroll certain patients or attempt to alter the enrollment order. This source of bias can be mitigated through high quality randomization generation, implementation, and allocation concealment procedures; the use of technology aids the implementation of such procedures.

We define allocation concealment as the component of blinding that prevents the treatment allocation for each patient from being revealed until the patient has been entered into the trial. This is distinct from treatment blinding which refers to what happens after randomization and which will be covered later. Efforts made to generate unpredictable and unbiased sequences are likely to be ineffective if those sequences are not adequately protected by allocation concealment. Those involved in the enrollment and assignment of participants must be kept unaware of the randomization sequence to avoid bias.

The Cochrane handbook ${ }^{3}$ provides a summary on the evidence as to whether the concealment of allocation sequence is associated with magnitude of effect estimates in controlled clinical trials. A pooled analysis of seven methodological studies found that effect estimates from trials with inadequate concealment of allocation or unclear reporting of the technique used for concealment of allocation were on average $18 \%$ (95\% confidence interval $5 \%$ to $29 \%$ ) more beneficial than effect estimates from trials with adequate concealment of allocation. ${ }^{4}$ A recent detailed analysis of three of these data sets combined (1346 trials from 146 meta-analyses) provides more information. Intervention effect estimates were exaggerated when there was inadequate allocation concealment in trials where a subjective outcome was analyzed, but there was little evidence of bias in trials with objective outcomes. ${ }^{5}$

Zhao et $a l^{6}$ describe the Captopril Prevention Project (CAPP) trial, which is an example of how selection bias can occur when technology is not used for the implementation of randomization. The purpose of the CAPP trial was to compare the treatment of hypertension by an angiotensin-convertingenzyme inhibitor (captopril) with the conventional therapy. Sealed and sequentially numbered envelopes were used to randomize subjects to the two treatment groups in the trial. However, when the data were analyzed, it was found that the treatment groups had small differences in some of the covariates recorded at randomization, including height, weight, and blood pressure. Although small, these differences had a significant effect on the results of the trial, causing the likelihood of stroke to appear greater in the captopril group. The fact that these differences in the treatment group occurred, combined with reports of violations in the randomization process at certain sites, indicates that there was likely to have been multiple instances of selection bias, thought to have been due to the use of envelopes to randomize subjects. Berger ${ }^{7}$ gives a full account of selection bias with further detailed examples.

Perhaps the most common use of technology in avoiding selection bias is to implement central randomization in open-label trials; this is encouraged in regulatory guidance. ${ }^{1}$ If technology is not used, then investigators will be aware of past allocations at their site and may thus have some success in guessing upcoming treatment allocations even if an appropriate allocation concealment procedure is used. Central randomization is effected by the investigator accessing the randomization system via a web interface or a telephone interactive voice response system (IVRS). The investigator inputs the patient characteristics into the system and the randomization details are returned - in an open-label trial, the details would include the allocated treatment. The central randomization may be stratified, but it is usually advisable not to stratify by site, as otherwise the investigator may have some success in guessing upcoming treatment allocations based on their knowledge of previous treatments; the predictability would be worse if a small block size relative to the number of treatments is used. 
If technology is not used for randomization in open-label trials then the allocation at site in multicenter trials will tend to be inadequate. The Cochrane handbook ${ }^{3}$ describes possible procedures that are inadequate including allocation by odd or even date of birth, or by some rule based on date of admission or hospital record number, etc. Alternatively, a randomization schedule may be generated, but this is still open to selection bias if there is some degree of control at the site level.

Even if the trial is double-blind, there may still be issues with selection bias if technology is not used. One way of organizing the randomization would be to send blocks of sequential numbered medication packs to sites and then instruct the investigator to allocate patients to treatment groups by allocating the next available pack number in the sequence. One problem with this approach is that the manual selection of packs is prone to error. The major issue, however, is that often the block size that is chosen will be fairly small, either for reasons of logistics/costs or a concern over potential compromise to the overall study balance. If the cost of medication is high, the trial sponsors will not want to ship blocks that are large to centers that may fail to recruit many, or indeed any, patients. If there are many centers, then there is also an increased motivation to keep the block size low to avoid an imbalance at the overall study level from potentially many incomplete blocks at the end of the trial. Usually the study power would not be severely compromised from the likely magnitude of study level imbalances that result in this manner but nevertheless it may seem undesirable to sponsors for other reasons. The danger of a small block size is that the investigator may be able to make some inference about the block size and past allocations from observation of pharmacological effects on the patients; this increases the risk of selection bias.

Whatever the reason, a number of articles have noted the prevalence of small block sizes in site-stratified trials. Hewitt and Torgerson ${ }^{8}$ summarized randomization methods reported in four major journals in 2002. Eighty-eight trials that gave sufficient detail reported using a restricted blocked randomization list (of the other studies reported, 17 were performed using minimization and 21 were performed using simple unconstrained randomization). Of the 88 trials that used a restricted randomization list for a trial comparing two treatments allocated in an equal ratio, 46 reported using a block size of less than six and 35 reported a block size of less than four. McEntegart ${ }^{9}$ reported on 40 double blind trials comparing two treatments that were stratified by site; the block sizes used were two $(15 \%)$, four $(70 \%)$, six $(10 \%)$, and mixed sizes $(5 \%)$.

The strategy of using site stratification together with small block sizes has more advantages for trial supplies and study level balance in trials of many treatments; it could be argued that the opportunities for selection bias are reduced in such trials relative to trials with fewer treatments. As an alternative, technology enables the use of a novel randomization technique that reduces the possibility of selection bias in trials of many treatments (Figure 1). ${ }^{10}$

This technique requires a blocked list of medication (pack) numbers, arranged in ascending number order. A separate blocked, unstratified randomization list is used to randomize patients into the trial. The medication is distributed to centers in sequential pack list order but not necessarily in complete blocks. This means that medication corresponding to all possible treatments may not be available at the center. When a patient is randomized they are allocated the next available randomization entry that corresponds to a treatment which is currently available at that center. In order to do this it may be necessary to skip over randomization entries where the medication for the corresponding treatment is not available. At each randomization the available entries will be looked at in sequence and any that have been skipped previously will be back filled.

In a standard centrally randomized trial, stock is replenished based on the quantity available per pack type, which for trials with many treatments results in large amounts of medication being stored at centers. Unlike the standard method, sending a quantity of packs sequentially means that the stock at centers will be resupplied based on the total number of packs available, regardless of pack type.

This randomization technique not only reduces medication wastage but also decreases the risk of selection bias as, due to incomplete blocks being sent, assumptions cannot be made about the allocation of the next treatment based on medication supply. Stratification factors such as gender and country could also be incorporated into this method. However, as a forced randomization occurs when medication is not available at the center for all possible treatments during randomization, this method will inevitably involve large numbers of forcing. In a Phase II study this is not likely to be a problem, but for a Phase III study this would need to be justified to regulators as ICH E9 Guidance requires that:

the next subject to be randomized into a trial should always receive the treatment corresponding to the next free number in the appropriate randomization schedule (in the respective stratum, if randomization is stratified). ${ }^{1}$

\section{Accidental bias}

Randomization tends to produce treatment groups in which the distributions of prognostic factors, known and unknown, 


\begin{tabular}{|c|c|c|c|}
\hline \multicolumn{5}{|c|}{ Packs table } \\
\hline Block & Pack number & Pack type & Shipped \\
\hline 1 & 1001 & B & Site 1 \\
\hline 1 & 1002 & A & Site 1 \\
\hline 1 & 1003 & C & Site 1 \\
\hline 1 & 1004 & D & Site 2 \\
\hline 2 & 1005 & D & Site 2 \\
\hline 2 & 1006 & B & Site 2 \\
\hline 2 & 1007 & A & Site 2 \\
\hline 2 & 1008 & C & Site 2 \\
\hline 3 & 1009 & A & Site 2 \\
\hline 3 & 1010 & B & \\
\hline 3 & 1011 & D & \\
\hline 3 & 1012 & C & \\
\hline
\end{tabular}

Randomization table

\begin{tabular}{|c|c|c|c|}
\hline Block & $\begin{array}{c}\text { Randomization } \\
\text { number }\end{array}$ & Treatment type & Allocated \\
\hline 1 & 001 & D & Patient 2 \\
\hline 1 & 002 & B & Patient 1 \\
\hline 1 & 003 & A & Patient 3 \\
\hline 1 & 004 & C & Patient 4 \\
\hline 2 & 005 & B & Patient 6 \\
\hline 2 & 006 & C & Patient 5 \\
\hline 2 & 007 & A & \\
\hline 2 & 008 & D & \\
\hline 3 & 009 & B & \\
\hline 3 & 010 & A & \\
\hline 3 & 011 & C & \\
\hline 3 & 012 & D & \\
\hline
\end{tabular}
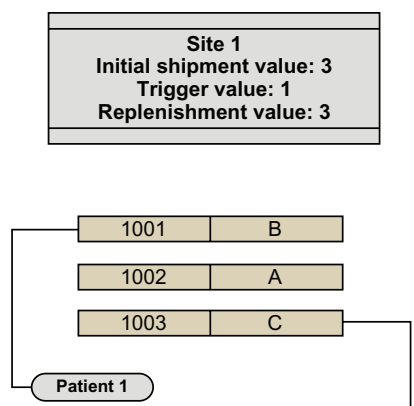

1. The first patient randomized into the study is at site 1 . There are no treatments on site that correspond to the treatment group assigned to randomization number 001 , so patient 1 is allocated randomization number 002 and pack 1001

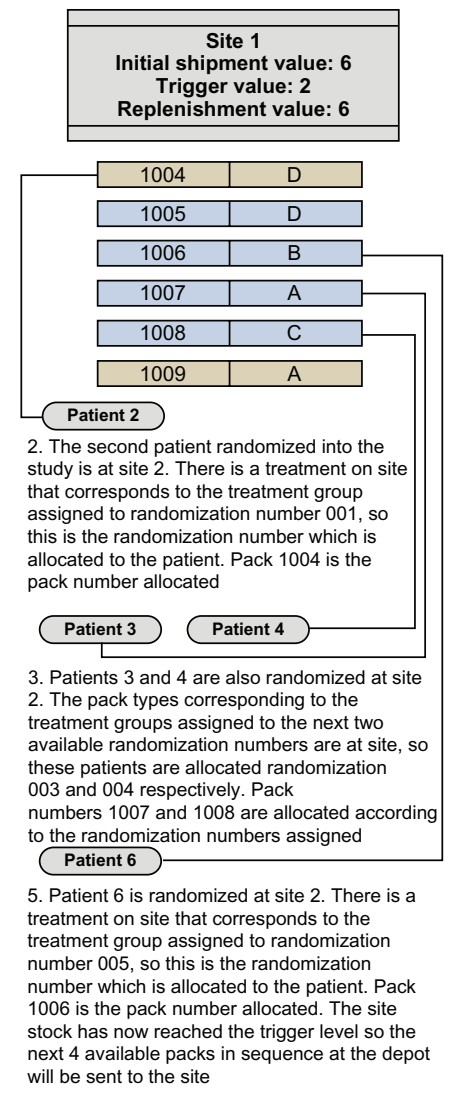

Figure I Randomization dependent on the treatments available at site.

are similar; however, individual trials are subject to chance imbalances. Whilst imbalances in strong and known factors can generally be adjusted for in the analysis, occasionally the imbalance may be so strong that, according to European regulators, "no adjustment may be sufficiently convincing to restore the reliability of the results." ${ }^{11}$ It has previously been suggested that use of a stratified randomization list or balancing using a dynamic randomization technique is a low-cost insurance policy against severe imbalance, given that most multicenter clinical trials are currently implemented using some form of technology. ${ }^{12}$ The use of electronic randomization is essential in trials using a dynamic randomization technique such as minimization. It is difficult to implement error free stratification in trials using randomization lists that are not managed electronically; in double blind trials the most common method is to provide patient numbered packs for each stratum which is wasteful of medication and prone to the investigator picking the wrong pack.

The reasons why electronic technology is used to implement clinical trials usually include factors other than the desire for electronic randomization (eg, electronic capture of data, costs savings on medication supplies, facilitation of competitive recruitment, and real time tracking of recruitment).
The extra cost of using stratification or dynamic randomization as opposed to unstratified randomization can be viewed as a marginal cost which is usually quite small relative to the costs of the overall system and total budget for the trial costs.

\section{Assessment bias through code breaking}

Maintaining the blind after treatment allocation is one of the items on the CONSORT checklist. ${ }^{2}$ If the blind is broken for one or more subjects during the trial then those patients may be subject to assessment bias. Knowledge of the patient's treatment may influence the investigator's assessments of the patient. One source of unblinding is through individual code breaks which occur for a variety of reasons including when there are perceived emergencies.

The ICH E9 Guidance states that breaking the blind for a single subject should be considered only when the knowledge of the treatment assignment is deemed essential by the subject's physician for the subject's care. Ayala and MacKillop ${ }^{13}$ describe the motivations for investigators to break the blind even if the information obtained is not likely to have a significant effect on the clinical management of the subject - for instance, they may feel embarrassed about referring the subject on to another doctor without knowing the treatment 
or they may feel uncomfortable discussing an adverse event with the subject.

The authors describe the potential consequences of breaking the blind for one subject. One consequence is the potential exclusion of the patient from the analyses. Even if, however, the patient is not excluded from all analyses, the assessments used in the analysis may have been affected by the investigator's knowledge of the treatment received. If the trial is site stratified, then knowing the treatment for one subject gives the investigator some information about the other treatments in the block, thus potentially opening on-going patients to assessment bias and future patients to selection bias.

There are two ways in which technology can help prevent unnecessary breaking of the blind:

- Investigators should be provided information about adverse events that might occur and the treatment required. As the trial progresses and more information is gathered, this information should be frequently updatedthe best way to do this is via a secure portal that houses all relevant information and documents.

- The IVRS or web interface that provides the emergency code break could have an automated, appropriate advisory script that cautions the investigator to only break the blind in a genuine emergency. The script could offer the chance to automatically connect to and speak to an on call physician. These protections would not be possible with traditional code breaker envelopes.

Furthermore, the availability of a controlled auditable process deters those who would wish to subvert the process.

Schulz and Grimes ${ }^{14}$ detail the efforts that investigators have made in order to decipher non-electronic randomization schemes (eg, they describe investigators holding up sequentially numbered, opaque, sealed envelopes to a special light bulb and late-night "ransacking" of the office files of the principal investigator for the allocation list). A more common problem is that investigators just open the sealed envelopes without good cause.

\section{Observer bias}

Another way in which technology can help avoid bias is in the prevention of observer bias. In trials which are driven by events such as myocardial infarctions or strokes there are usually some criteria as to what constitutes an event. The gold standard is to have events submitted to a panel of expert adjudicators who are blind to the treatment groups. In many modern clinical trials the timely completion of adjudications would be regularly challenged by the logistics of performing these reviews in a paper environment. A lengthy adjudication process limits the ability for sponsors to make timely decisions based upon review committee findings. Web-based applications are available to facilitate central management of adjudication activities as discussed in Nolen et al. ${ }^{15}$

When an event or endpoint occurs for a subject, relevant clinical documents are collected in the system to create an electronic dossier. Once a dossier is complete, the system enables reviewers to analyze the appropriate dossier and record their assessments, with built-in edit checks to ensure collection of clean data at the time of entry. The completed reviewers' assessments are compared by the system, and if discordance is found, the dossier is automatically reassigned for tiebreaker review. Workflow and electronic query tools available within such systems streamline the review process enabling the process to be highly efficient.

The reason why adjudication is needed is demonstrated in a trial of patients with multiple sclerosis. ${ }^{16}$ Participants were examined by two groups of neurologists, one group being blinded and the other unblinded. The former found no effect of treatment, while the latter found evidence of efficacy.

\section{Operational bias in adaptive trials}

Another example where using an electronic system to centrally randomize can reduce bias is when there is expected to be a mid-study modification, either to alter the allocation ratio in which treatments are assigned or even to drop or add to the set of treatments being randomized. There are two main reasons why it is necessary to use an electronic system.

The first is that it is desirable for site personnel to be unaware of the precise time of any design adaptation. Knowledge of the adaption may influence subject selection and introduce bias into the sample, both before and after the design adaptation is made. Sites may be more likely to enter certain subjects into the trial if they are aware that suboptimal treatments have been eliminated as a result of an adaptation. Conversely, if site personnel are expecting an adaption to take place in the near future, they may be reluctant to randomize subjects prior to the adaption. This topic is addressed by the current regulatory guidance in this area. For instance the European Guidance ${ }^{17}$ discusses two scenarios where knowledge of the fact that an adaption has taken place may lead to investigators being more or less willing to enter certain types of patients. Further to this, the Food and Drug Administration 
Draft Guidance ${ }^{18}$ is more specific and addresses the risk of treatment bias, stating that:

knowledge of the interim unblinded data used to make the adaption decision, or even knowledge only of the specific adaptive choice, has the potential to introduce operational bias into the treatment-effect estimates. ${ }^{18}$

Technology can be used to switch or alter the randomization method behind the scenes without any site or key study personnel being aware of the switch. For instance the IVRS/ web system would be set up to allow one of a number of preplanned switches such as dropping a treatment arm to be automatically implemented in real time upon receiving an IVRS/web transaction call from a member of an independent Data Monitoring Committee.

The second reason to use technology is to ensure that randomization changes are applied correctly and without errors. For example if it is wished to drop a treatment in a non-automated trial where allocation is made by assigning patient and pack numbers sequentially, the trial leader could ask site personnel to skip certain patient/pack numbers (these will correspond to the randomization list if the traditional method of pack allocation is used); this would be prone to introducing human error into the randomization procedure. The skipping pattern may also enable the investigator to deduce the randomization block size and hence increase their ability to infer the treatment allocations of current and future subjects which would increase the risk of selection bias.

Approaches to managing mid-study changes to randomization using electronic systems are described by Byrom et al. ${ }^{19}$

\section{Assessment and operational bias due to dose adjustments}

In blinded clinical trials where the medication that is given needs to be adjusted according to recorded observations, a potential problem can arise: if certain observations are only associated with one of the treatment groups, how can the study be kept blinded to those involved? Sackett provides some examples and notes that creativity will be needed in implementing such trials. ${ }^{20}$

Consider the example of stroke and cardiovascular studies where a placebo or comparator group is compared to warfarin or a combination including warfarin. Warfarin is an anticoagulant so for patient safety reasons its blood thinning effects must be measured at regular intervals. Based on the reading from the blood sample, the patient's dose can be adjusted accordingly. The reading indicates the blood clotting tendency and is measured using prothrombin time, which is commonly represented as an international normalized ratio (INR) value. INR values can be measured using a point-of-care (PoC) device that can provide an instant INR estimate using only a single drop of blood. However, in order to maintain the blind, a process is needed to ensure that site personnel cannot use this reading to identify the patients that are taking warfarin. This can be achieved with the assistance of an unblinded site investigator. For example, in the Warfarin Aspirin Symptomatic Intracranial Disease trial, the unblinded investigator had no role in patient care other than to determine the dose of active or placebo warfarin. ${ }^{21}$ Results of INRs were faxed to the unblinded investigator and were not to be communicated to site staff. Clearly, the methodology is more secure if an encryption system is used, which has been shown to be the case in several trials. Using an IVRS provider to manage the reading in a blinded manner will allow sites to have access to the important information they need for safely running the trial without revealing to them which treatment a patient is on.

In order to allow the INR values to be managed by the IVRS provider, the PoC device is modified so that, instead of displaying the actual INR value, the output is given in the form of an encrypted code. This encrypted code can then be input into the IVRS via a call from the site personnel, who will then be provided with a reading by the IVRS. This reading could either be the true INR value recorded or, if the subject is on placebo, a masked value (Figure 2).

When using this method, one of the main challenges is allocating the masked values in a manner that does not allow placebo patients to be identified due to a predictable pattern. This is done by mimicking the values that would be seen for a patient receiving the experimental treatment as closely as possible.

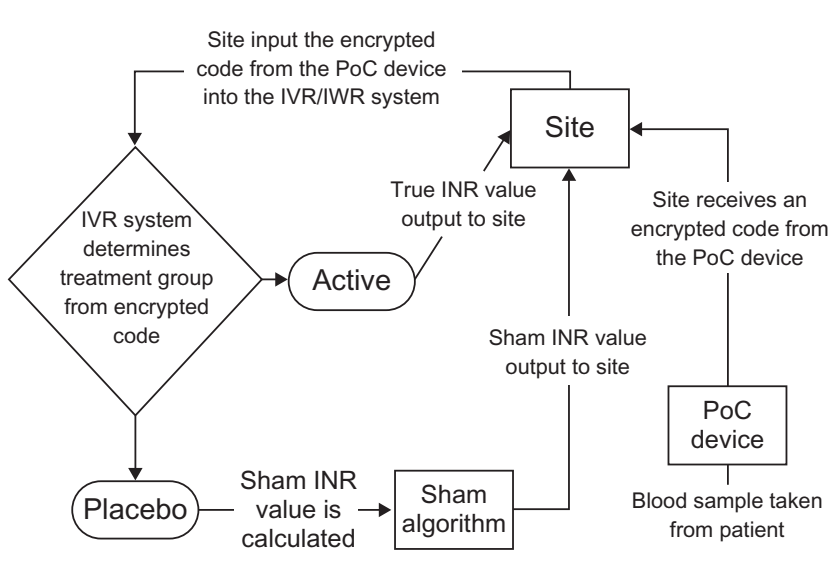

Figure 2 Process for managing blinded INR values.

Abbreviations: INR, international normalized ratio; IVR, interactive voice response; IWR, interactive web response; PoC, point-of-care. 
There are several ways of producing masked INR values. One of the simplest options is to randomly select a value from a known distribution of INR values which have been identified from previous data. However, the set of results that this method would produce for a placebo patient would not correlate to the expected effect of warfarin treatment over a period of time, which is potentially unblinding. This method can be improved upon by taking into account the point in the treatment cycle where the visit is being made. Randomly selecting from a known distribution of samples recorded on the same treatment day as the visit the patient is currently on improves the plausibility of the mock reading that is given. However, the overall sequence of values for the patient would not necessarily be consistent. An alternative method is to assign a sequence of plausible values for placebo patients, where mock readings are then selected in sequence from the predefined list of values. Each sequence would be based on values obtained through previous research and there would need to be a sufficiently large number of sequences to avoid patterns being identified.

However, these methods do not take into account scenarios where the site would expect to see a specific change in readings such as after dose adjustments or when visits have been missed. In order to incorporate a full range of factors including age, gender, previous dose, and previous INR values, a sham algorithm is needed. A sham algorithm uses these factors to model a plausible mock INR value for the patient based on trends recorded from previous studies. A sham algorithm was implemented by an IVRS in the ROCKET study. ${ }^{22}$

An alternative method for implementing dose adjustments that does not require sampling from the results of previous trials is to use a dynamic algorithm. The algorithm is dynamic in that the data used is not known prior to the start of the trial. The process used for this algorithm is described below:

- Every time there is a decision for a patient taking the experimental treatment, a copy of that decision is made in a system table that will determine all decisions made for patients in the placebo group. The copied record is marked as "available."

- At the point at which a decision needs to be made for a patient in the placebo group, the system chooses an entry at random from the above described table. The chosen entry is marked as "used" and is not available for further allocation.

- As the table for decision making for the placebo group is continuously compiled based on experience in the experimental group, it ensures a degree of decision matching that is sufficient to maintain the blind. Even if the pattern of allocations alters through the study, this matching design will ensure that the altering pattern is reflected in the placebo allocations.

- In case there are more patients on placebo than on the experimental compound, then it is prudent to have some default records that are used to supplement the table if necessary. The composition of the default records could be altered in the light of experience.

- Refinements to the method are possible; for instance, separate sections of the table could be made for different study visits. Similarly, stratification can be incorporated if felt appropriate.

The method of producing mock INR values that is used depends on the preference of the study team and the chosen technique can be applied by an IVRS. Warfarin studies are not the only example in which these techniques can be used. In fact, any study in which observations could be associated with treatment given can apply this method to minimize the risk of unblinding.

\section{Results and discussion}

In this article, we have described the role of technology in reducing bias in the execution of clinical trials. It should be acknowledged, however, that technology can increase the risk of bias if it is not implemented carefully.

In current randomization and trial supply management systems it is normal practice to separate the randomization step from the dispensing step. ${ }^{23,24}$ Then, any pack of the appropriate treatment can be dispensed to any patient randomized to that treatment. This allows supply savings compared to traditional methods as packs for withdrawn patients are not wasted and supplies can be directed to the sites with the highest recruitment focus. The delinking of the randomization list and packaging list in electronic randomization systems together with the system inventory management policy of not sending too many resupplies to sites means there are various sources of unblinding if care is not taken:

- There is always the chance of running out of the required pack type for randomization. Then the question is what to do - either halt the call or web transaction which means there is an opportunity for the investigator to make logical deductions about the stock at the site, or to allow the system to force the randomization from the subsets of treatments for which packs are available at site. ${ }^{25}$ Of course, the best approach is to have a well-planned strategy that ensures this is a rare event.

- The chance of unblinding through what we have termed "dispensing order unblinding" or "pack separation" if 
care is not taken. The way to avoid this is to have random selection of packs at the site and if possible a scrambled or double-randomized pack list where there is no association between the pack number and its position in the packaging list. ${ }^{26}$

- There is a risk of unblinding if consignments are sent that only contain medication for one subject. However, the addition of packs to blind the consignment can cause unnecessary wastage. This can be mitigated by using blind group ordering which is based on the principle that the initial supply to the site contains the random blinding medication, rather than having to add it to each consignment. The stock quantity levels that cause a resupply when they are breached (trigger levels) are based on total stock as well as or instead of trigger levels for individual pack types. This means that when a pack is used, the consignment raised to replace it may be replacing the random kit at the site and not necessarily the pack that was just used, so the single pack consignment is not partially unblinding. ${ }^{27}$

- The site may be able to deduce the block size if blocks of randomization code are not allocated to the site in a site-stratified trial until they are needed and the randomization number is revealed to the site investigator as confirmation of the randomization transaction. ${ }^{28}$ This practice of on-demand blocks has been termed dynamic allocation of blocks. ${ }^{12,28}$ It allows for easy addition of previously unplanned sites to boost recruitment. If, however, the randomization number is revealed to the investigator then he/she can deduce the block size from the step change in the randomization numbers when an allocation is made from a new block. If it is felt necessary to utilize a randomization number as an identifier, then a scrambled number that differs from the sequence number on the randomization list should be used.

\section{Conclusion}

To summarize, due to the risks that bias poses to the credibility of clinical trial results, the literature reviewed would suggest that it is preferable to use technology in clinical trials as bias can be caused by using some of the alternative, and often inadequate, randomization and blinding techniques referred to in this article. Although the use of technology can generally help to reduce bias compared to traditional methods of randomization and medication management, in trials where technology has not been used effectively or when due consideration has not been given to potential unblinding scenarios, bias can still be a potential issue. However, because of the flexibility and innovation in clinical trial design that is enabled by the use of technology, the benefits should outweigh the unblinding risks associated with separating the dispensing and randomization as long as appropriate attention has been given to maintaining the blind.

\section{Disclosure}

The authors are employed by Perceptive Informatics Inc, Nottingham, UK, a provider of eClinical technology services, including those described in the article; however, no funding or payment was received in association with this work.

\section{References}

1. US Department of Health and Human Services. Guidance for Industry E9 Statistical Principles for Clinical Trials. Rockville, Food and Drug Administration, 1998. Available from: http://www.fda.gov/ downloads/Drugs/GuidanceComplianceRegulatoryInformation/ Guidances/UCM073137.pdf. Accessed November 23, 2012.

2. Moher D, Hopewell S, Schulz KF, et al. CONSORT 2010 explanation and elaboration: updated guidelines for reporting parallel group randomized trials. BMJ. 2010;340:c869.

3. Higgins JPT, Green S, editors. Cochrane Handbook for Systematic Reviews of Interventions Version 5.1.0 [updated Mar 2011]. The Cochrane Collaboration; 2011. Available from: http://www.cochranehandbook.org. Accessed November 23, 2012.

4. Pildal J, Hróbjartsson A, Jørgensen KJ, Hilden J, Altman DG, Gøtzsche PC. Impact of allocation concealment on conclusions drawn from meta-analyses of randomized trials. Int J Epidemiol. 2007;36(4): $847-857$.

5. Wood L, Egger M, Gluud LL, et al. Empirical evidence of bias in treatment effect estimates in controlled trials with different interventions and outcomes: meta-epidemiological study. BMJ. 2008;336(7644):601-605.

6. Zhao W, Hill MD, Palesch Y. Minimal sufficient balance - a new strategy to balance baseline covariates and preserve randomness of treatment allocation. Stat Methods Med Res. January 26, 2012. Epub ahead of print.

7. Berger VW. Selection Bias and Covariate Imbalances in Clinical Trials. New York: John Wiley \& Sons; 2005.

8. Hewitt CE, Torgerson DJ. Is restricted randomisation necessary? BMJ. 2006;332(7556):1506-1508.

9. McEntegart D. A relevant question for site balanced studies relates to the comparative predictability of minimization to the alternative of permuted blocks [letter to the editor]. Contemp Clin Trials. 2010;31(6):507.

10. Morrissey M, McEntegart D, Lang M. Randomisation in double-blind multicentre trials with many treatments. Contemp Clin Trials. 2010; 31(4):381-391.

11. Committee for Proprietary Medicinal Products. Points to Consider on Adjustment for Baseline Covariates. The European Agency for the Evaluation of Medicinal Products; 2003. Available from: http:// www.emea.europa.eu/pdfs/human/ewp/286399en.pdf. Accessed November 23, 2012

12. McEntegart DJ. The pursuit of balance using stratified and dynamic randomisation techniques. Drug Inf J. 2003;37:293-308.

13. MacKillop N, Ayala E. Educating investigators to understand when to break the blind. Applied Clinical Trials Online. Santa Monica, CA: Advanstar Communications; 2001. Available from: http://www. appliedclinicaltrialsonline.com/appliedclinicaltrials/Feature+Article/ Educating-Investigators-to-Understand-When-to-Brea/ArticleStandard/ Article/detail/90057. Accessed November 23, 2012.

14. Schulz KF, Grimes DA. Allocation concealment in randomised trials: defending against deciphering. Lancet. 2002;359(9306):614-618. 
15. Nolen TL, Dimmick BF, Ostrosky-Zeichner L, et al. A web-based endpoint adjudication system for interim analyses in clinical trials. Clin Trials. 2009;6(1):60-66.

16. Noseworthy JH, Ebers GC, Vandervoort MK, Farquhar RE, Yetisir E, Roberts R. The impact of blinding on the results of a randomized, placebo-controlled multiple sclerosis clinical trial. Neurology. 1994; 44(1):16-20.

17. Committee for Medicinal Products for Human Use (CHMP). Reflection Paper on Methodological Issues in Confirmatory Clinical Trials Planned with an Adaptive Design. London: European Medicines Agency; 2007. Available from: http://www.ema.europa.eu/docs/en_GB/ document_library/Scientific_guideline/2009/09/WC500003616.pdf. Accessed November 23, 2012.

18. Food and Drug Administration. Guidance for Industry: Adaptive Design Clinical Trials for Drugs and Biologics [draft guidance]. Rockville, MD: US Food and Drug Administration; 2010. Available from: http:// www.fda.gov/downloads/Drugs/GuidanceComplianceRegulatoryInfo rmation/Guidances/ucm201790.pdf. Accessed November 23, 2012.

19. Byrom B, McEntegart D, Nicholls G. Adaptive infrastructure. In: Pong A, Chow S-C, editors. Handbook of Adaptive Designs in Pharmaceutical and Clinical Development. London: Taylor \& Francis Group; 2010:125.

20. Sackett DL. Clinician-trialist rounds: 6 . Testing for blindness at the end of your trial is a mug's game. Clin Trials. 2011;8(5):674-676.

21. Warfarin-Aspirin Symptomatic Intracranial (WASID) Tria Investigators. Design, progress and challenges of a double-blind trial of warfarin versus aspirin for symptomatic intracranial arterial stenosis. Neuroepidemiology. 2003;22(2):106-117.
22. ROCKET AF Study Investigators. Rivaroxaban-once daily, oral, direct factor Xa inhibition compared with vitamin $\mathrm{K}$ antagonism for prevention of stroke and Embolism Trial in Atrial Fibrillation: rationale and design of the ROCKET AF study. Am Heart J. 2010;159(3):340-347.

23. Byrom B. Using IVRS in clinical trial management. Applied Clinical Trials. Oct 2002. Available from: http://www.clinphone.com/files/ item132.aspx. Accessed November 23, 2012.

24. Dowlman N. Efficiency on demand. European Pharmaceutical Contractor. Jun 2009. Samedan Ltd; 2009. Available from: http:// www.perceptive.com/library/published-papers/2009/. Accessed November 23, 2012.

25. McEntegart D. Forced Randomization: when using interactive voice response systems. ACT. 2003;12(10):50-58. http://www.clinphone. com/files/item91.aspx. Accessed November 23, 2012

26. Lang M, Wood R, McEntegart D. Protecting the blind. GCPj. Nottingham: T\&F Informa UK Ltd; 2005. Available from: http://www.perceptive.com/index.php/download_file/view/151/.Accessed November 23, 2012.

27. Waters S, Dowlman I, Drake K, Gamble L, Lang M, McEntegart D. Enhancing control of the medication supply chain in clinical trials managed by interactive voice response systems. Drug Inf J. 2010;44(6):727-740. Available from: http://www.nxtbook.com/ nxtbooks/dia/druginformationjournal1110/index.php\#/0. Accessed November 23, 2012

28. McEntegart D. Blocked randomization. In: D’Agostino R, Sullivan L, Massaro J, editors. Wiley Encyclopedia of Clinical Trials. Hoboken: John Wiley \& Sons, Inc; 2008.
Open Access Journal of Clinical Trials

\section{Publish your work in this journal}

The Open Access Journal of Clinical Trials is an international, peerreviewed, open access journal publishing original research, reports, editorials, reviews and commentaries on all aspects of clinical trial design, management, legal, ethical and regulatory issues, case record form design, data collection, quality assurance and data auditing

\section{Dovepress}

methodologies. The manuscript management system is completely online and includes a very quick and fair peer-review system, which is all easy to use. Visit http://www.dovepress.com/testimonials.php to read real quotes from published authors. 